An earlier study demonstrated a significantly decreased level of CD103+CD4+/CD4+ in radiographic stage I sarcoidosis compared with healthy controls and significantly elevated levels in radiographic stage II/III sarcoidosis [5]. We found no significant differences in CD103+CD4+/CD4+ levels between the two radiographically staged groups in our study.

s-ACE is an important marker of sarcoidosis with high sensitivity but low specificity. A polymorphism in the ACE gene affects normal s-ACE concentration [8]. A homozygotic deletion in the promoter resulted in high levels of s-ACE (59.8 versus $32.2 \mathrm{U} \cdot \mathrm{L}^{-1}$ for the homozygous insertion allele; $\mathrm{p}<0.0001$ ) in a north German population [8]. Screening for this polymorphism is not common practice; however, the use of genotype-corrected normal ranges may increase the value of this parameter in the diagnosis and follow-up of sarcoidosis.

The combinations of CD103+CD4+/CD4+, CD4+/CD8+ and lymphocyte percentages that were tested in this study showed limited diagnostic value in this unselected group of sarcoidosis patients. Previously proposed criteria based on combinations of CD103+CD4+/CD4+ and CD4+/CD8+ had a lower sensitivity in this study than previously reported. s-ACE is an established marker of sarcoidosis and is the most useful of the markers that were investigated in this study.

Sarcoidosis is a disease with several phenotypes, which complicates the search for useful diagnostic markers. There remains an unmet need for new improved methods to support diagnosis when there is suspicion of sarcoidosis.

\section{Hyldgaard, S. Kaae, M. Riddervold, H.J. Hoffmann and O. Hilberg \\ Dept of Respiratory Medicine, Aarhus University Hospital,} Aarhus, Denmark.
Correspondence: C. Hyldgaard, Dept of Respiratory Medicine, Aarhus University Hospital, Norrebrogade 44, Aarhus 8000 C, Denmark. E-mail: charhyld@rm.dk

Statement of Interest: None declared.

\section{REFERENCES}

1 Statement on sarcoidosis. Joint Statement of the American Thoracic Society (ATS), the European Respiratory Society (ERS) and the World Association of Sarcoidosis and Other Granulomatous Disorders (WASOG) adopted by the ATS Board of Directors and by the ERS Executive Committee, February 1999. Am J Respir Crit Care Med 1999; 160: 736-755.

2 Kantrow SP, Meyer KC, Kidd P, et al. The CD4/CD8 ratio in BAL fluid is highly variable in sarcoidosis. Eur Respir J 1997; 10: 2716-2721.

3 Costabel U. CD4/CD8 ratios in bronchoalveolar lavage fluid: of value for diagnosing sarcoidosis? Eur Respir J 1997; 10: 2699-2700.

4 Welker L, Jorres RA, Costabel U, et al. Predictive value of BAL cell differentials in the diagnosis of interstitial lung diseases. Eur Respir J 2004; 24: 1000-1006.

5 Lohmeyer J, Friedrich J, Grimminger F, et al. Expression of mucosarelated integrin $\alpha^{\mathrm{E}} \beta_{7}$ on alveolar T cells in interstitial lung diseases. Clin Exp Immunol 1999; 116: 340-346.

6 Heron M, Slieker WA, Zanen P, et al. Evaluation of CD103 as a cellular marker for the diagnosis of pulmonary sarcoidosis. Clin Immunol 2008; 126: 338-344.

7 Kolopp-Sarda MN, Kohler C, De March AK, et al. Discriminative immunophenotype of bronchoalveolar lavage CD4 lymphocytes in sarcoidosis. Lab Invest 2000; 80: 1065-1069.

8 Ruprecht B, Schurmann M, Ziegenhagen MW, et al. Corrected normal values for serum ACE by genotyping the deletion-/ insertion-polymorphism of the ACE gene. Pneumologie 2001; 55: 326-332.

DOI: $10.1183 / 09031936.00144311$

\title{
Surgical salvage following stereotactic body radiotherapy for early-stage NSCLC
}

\section{To the Editors:}

For medically inoperable early-stage nonsmall cell lung cancer (NSCLC) patients, stereotactic body radiotherapy (SBRT) is the emerging treatment of choice. SBRT differs from conventional radiotherapy (RT) in that large doses of radiation are employed over a few (usually fewer than five, but sometimes up to 10) fractions to radioablate the tumour. Safe delivery of such profoundly damaging doses requires highly precise RT, in conjunction with image guidance and careful treatment planning.

Several prospective clinical trials have confirmed the feasibility, safety and efficacy of SBRT, consistently reporting local control rates of $80-95 \%$, comparable to surgical resection [1-3], although assessment of response is more complicated than it is following surgery [3]. Radiographic changes following SBRT differ from those following conventional RT, suggesting a unique histological response, and characterisation of these changes is a clinical challenge. Little is known of the histology of local recurrence following SBRT, since medically inoperable patients who fail locally cannot be salvaged surgically. Fineneedle aspiration biopsy provides only cytological information and is subject to false-negative results, while very frail patients may not even be able to tolerate the biopsy. Without histological confirmation, local failure (LF) is a clinical rather than a pathological diagnosis. In this report, we review our experience of four patients originally treated with SBRT who were salvaged surgically for their presumed LF.

From October 2004 to December 2010, we treated 209 patients with early (T1-2N0M0) NSCLC using SBRT on a prospective institution research ethics board-approved protocol. All patients were deemed medically inoperable by an experienced 
thoracic surgeon, typically due to medical comorbidities. Standard staging investigations included 2-fluoro-2-deoxy-Dglucose (FDG)-positron emission tomography (PET). Our SBRT technique has previously been described [4, 5]. Patients underwent post-treatment PET-computed tomography (CT) and serial CT scans as per our protocol.

Cases in which there was high clinical suspicion for LF were discussed at our weekly rounds. Further investigations (including repeat PET-CT and/or biopsy) were requested accordingly and cases were also presented for discussion at our multidisciplinary conference.

Of the 209 patients treated, we identified six cases with local progression (3-yr local control 94.6\%). In four of these instances, the local progression represented the only known site of disease. While all four of these patients had initially been deemed medically inoperable at the time of initial diagnosis, they were all considered fit for surgery at the time of presumed post-SBRT failure (table 1). The initial reasons for inoperability in these four patients included comorbidities, such as recent coronary event, cerebrovascular event, severe pulmonary disease or other medical illnesses such as diabetes. Improvement or stabilisation of comorbidities after SBRT eventually allowed these patients to undergo major pulmonary resection.

Although an initial reduction in the maximum standardised uptake value (SUV) of the tumour was seen in three out of these four patients after SBRT, the maximum response on CT never exceeded stable disease as per Response Evaluation Criteria in Solid Tumors (RECIST). In fact, at the 3-month mark, two patients already had growth of the index lesion that fulfilled the criteria for progressive disease. All patients had a growing mass lesion at the treated site on serial CT imaging, which prompted further investigations. Isolated LF was declared based on clinical and/or pathological evidence.
For patient A, there was a small initial regression on post-SBRT scans; however, the mass then grew to $4 \mathrm{~cm}$ before a positive biopsy. Patient B's tumour, conversely, demonstrated growth on imaging, but two biopsies returned negative before the third one revealed malignancy. Patient C's tumour grew until it was twice as large as the initially treated primary. Repeat PET was not carried out since the initial tumour was not FDGavid $(S U V=1.0)$ and the patient declined a biopsy. Patient D had a growing lesion on serial CT scans and on FDG-PET the SUV was more than double that of the incident lesion (8.9 versus 4.3 ).

Upon reassessment by the thoracic surgeon, these four patients were now felt to be medically operable. Major pulmonary resection, consisting of lobectomy and nodal sampling, was undertaken for attempted surgical salvage (table 2). Adhesions were found in all cases, and this was the most commonly described intraoperative finding attributable to the prior RT. Although in some cases these were simply dissected, they precluded the plan for a thoracoscopic resection in one case, requiring conversion into an open thoracotomy. In another patient, adhesion of lung to the overlying ribs required a partial chest wall resection. The duration of each surgery was within normal limits, and there were no significant intraoperative or post-operative complications.

Surgical pathology was re-reviewed by a single experienced thoracic pathologist. The resected tumour size was larger than the original lesion in each case with significant necrosis to varying degrees, reflecting at least a partial pathological response to the radiotherapy. In the three cases where viable cancer cells were found, the pathological subtype of residual NSCLC was identical to the originally treated lesion, although in the third case adenocarcinoma was located only in focal nests within the otherwise necrotic mass, accounting for only $5-10 \%$ of the resected tissue. Adequate surgical margins were achieved in all three of these cases. In the fourth case, the

TABLE 1 Initial patient and tumour characteristics, stereotactic body radiotherapy (SBRT) treatment and response

\begin{tabular}{|c|c|c|c|c|c|c|c|c|c|}
\hline Patient & $\begin{array}{l}\text { Age } \\
\text { yrs }\end{array}$ & $\begin{array}{c}\text { ECOG } \\
\text { PS }\end{array}$ & $\begin{array}{l}\text { Major comorbidity } \\
\text { preventing surgery }\end{array}$ & $\begin{array}{l}\text { Initial clinical } \\
\text { stage (size } \\
\text { in } \mathrm{cm})\end{array}$ & Histology & $\begin{array}{l}\text { Dose Gy/ } \\
\text { fractions } n\end{array}$ & $\begin{array}{c}\text { CT response } \\
\text { to SBRT at } \\
3 \text { months }\end{array}$ & $\begin{array}{l}\text { Pre-SBRT } \\
\text { SUV }\end{array}$ & $\begin{array}{l}\text { Post-SBRT } \\
\text { SUV }\end{array}$ \\
\hline
\end{tabular}

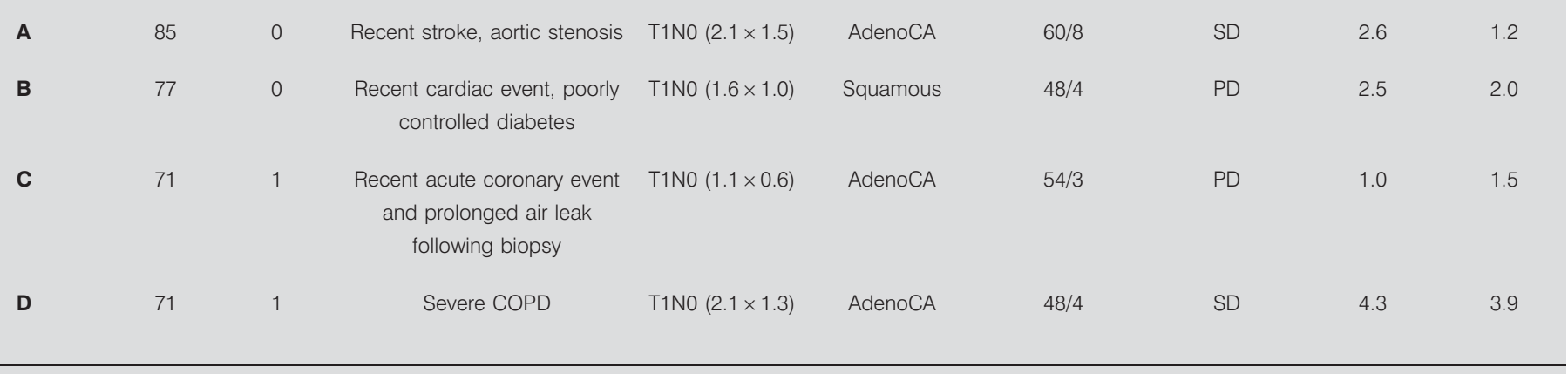

ECOG PS: Eastern Cooperative Oncology Group performance status; CT: computed tomography; AdenoCA: adenocarcinoma; SUV: standardised uptake value; SD: stable disease; PD: progressive disease; COPD: chronic obstructive pulmonary disease. 
resected mass simply demonstrated necrotic and fibrotic tissue with no evidence of viable cancer cells. There was evidence of mediastinal nodal disease in the second patient, requiring subsequent (conventional) mediastinal RT and adjuvant cisplatin-based chemotherapy. Microscopic evaluation of the normal tissue demonstrated some common responses to the ablative radiotherapy, including necrosis, interstitial fibrosis, diffuse alveolar damage and vascular changes including obliterative endarteritis, fibrointimal thickening and focal infarcts. All four patients remain alive with no evidence of disease, after a median follow-up of 30 months (range 1435 months) since their surgery.

Our experience confirms the results of several large prospective trials demonstrating excellent local control of early-stage NSCLC with SBRT. We have also shown that carefully selected patients who do experience LF are able to safely and successfully undergo post-SBRT resection without significant surgical morbidity. Medical operability is not always fixed and constant as shown in these four cases, and surgical salvage should not be dismissed offhand in patients previously felt to be medically inoperable.

Presently, we are unable to accurately characterise the radiological changes seen following SBRT [3], particularly at the site of treatment where marked inflammatory and fibrotic changes may resemble or obscure signs of recurrent disease [7]. Although a healthy level of clinical suspicion for recurrence should be maintained, false positives are possible (as in patient D) and the potential morbidity of unnecessary major surgery should be kept in mind.

The timeliness with which LF is identified are equally important. Patient B was closely monitored and investigated for several months prior to proceeding with surgery. Whether this delay in diagnosis had any clinical implication is uncertain. An earlier resection may potentially have caught the disease before it had spread to the mediastinal nodes.

At the other end of this spectrum, an adequate interval of time should be granted after SBRT prior to judging failure. Patient C's post-operative pathology report only revealed focal nests of adenocarcinoma within an otherwise necrotic mass. As it composed only $5-10 \%$ of the tumour volume, it would be difficult to attribute the doubling in tumour size observed on imaging to this low fraction of viable cancer alone. The natural course of these cancer cells, had they remained in situ, is unclear. Surgery was performed less than $1 \mathrm{yr}$ after completion of SBRT and it is possible that these residual cancer cells may not have remained viable over longer follow-up. We do not feel that the pathological findings indicate a clear case of LF.

For patient A, the surgery was undertaken in a relatively timely manner, and the tumour was resected without complications. The patient remains alive and well nearly 2 yrs later without evidence of disease.

Closely coordinated care and multidisciplinary meetings are essential when deciding upon surgical salvage. Serial CT scans are often obtained in routine follow-up. Particular attention should be paid to the exact location of the recurrence in relation to the original tumour and if possible correlated to the dosimetric data from the radiotherapy plan. PET-CT scans are 
also useful and we frequently make use of them for such cases, although as demonstrated in this and other series [7], they are not entirely reliable due to false positives, and their results should be considered carefully in light of other clinical and radiological findings.

The gold standard remains tissue confirmation from the suspicious mass. However, the sensitivity in the context of the extensive fibrotic and/or necrotic changes from the high-dose SBRT is not entirely known, and in the case of patient B, pathological confirmation of recurrence only occurred after a total of 11 passes had been taken over three separate biopsy attempts. The procedure itself may be relatively contraindicated in patients with poor pulmonary status. As a result, careful consideration should go into the selection and planning of such procedures.

We have demonstrated that local failures following SBRT may be successfully and safely salvaged, particularly in the case of isolated local failures. Two recent reports from Japan demonstrated surgical salvage in patients who were initially medically operable but had refused surgery up-front [8, 9]. Our series demonstrates that surgical salvage may also be feasible in selected patients that were initially considered medically inoperable. Our experience would suggest that the optimal surgical approach for these patients should remain thoracotomy as opposed to video-assisted thoracoscopic surgery. The challenge remains in identifying those patients who truly have local failure in a timely manner. Careful patient selection combined with surgical expertise allows for successful salvage with minimal morbidity. Once again, close and regular follow-up as well as close cooperation between the appropriate medical disciplines is emphasised.

\section{Z. Allibhai*, B.C.J. Cho*, M. Taremi", S. Atallah*, A. Hope*,} D. Hwang ", S. Keshavjee ${ }^{+}$, M. Tsao ${ }^{\oplus}$, K. Yasufuku ${ }^{+}$, S-W. Kim* and A. Bezjak*

*Dept of Radiation Oncology, Princess Margaret Hospital, "Dept of Pathology, and, 'Division of Thoracic Surgery, Toronto General Hospital, Toronto, and "Dept of Radiation Oncology, Stronach Regional Cancer Centre, Newmarket, ON, Canada.
Correspondence: Z. Allibhai, Dept of Radiation Oncology, Princess Margaret Hospital, 620 University Avenue, Toronto, ON, M5G 2M9, Canada. E-mail: zishan.allibhai@gmail.com

Statement of Interest: A statement of interest for this study can be found at www.erj.ersjournals.com/site/misc/statements. xhtml

Acknowledgements: We would like to personally thank C. Torres (Dept of Radioation Oncology, Princess Margaret Hospital, Toronto, ON, Canada) for her dedication and efforts in data collection as well as her other contributions towards our SBRT clinical and research programme.

\section{REFERENCES}

1 Timmerman R, Paulus R, Galvin J, et al. Stereotactic body radiation therapy for inoperable early stage lung cancer. JAMA 2010; 303: 1070-1076.

2 Chi A, Liao Z, Nguyen NP, et al. Systemic review of the patterns of failure following stereotactic body radiation therapy in early-stage non-small-cell lung cancer: clinical implications. Radiother Oncol 2010; 94: 1-11.

3 Dahele M, Palma D, Lagerwaard F, et al. Radiologic changes after stereotactic radiotherapy for stage I lung cancer. J Thorac Oncol 2011; 6: 1221-1228.

4 Grills IS, Mangona VS, Welsh R, et al. Outcomes after stereotactic lung radiotherapy or wedge resection for stage I non-small cell lung cancer (NSCLC). J Clin Oncol 2010; 28: 928-935.

5 Dahele M, Pearson S, Purdie T, et al. Practical considerations arising from the implementation of lung stereotactic body radiation therapy (SBRT) at a comprehensive cancer centre. J Thorac Oncol 2008; 3: 1332-1341.

6 American Joint Committee on Cancer. Cancer Staging Manual. 7th Edn. Chicago, American Joint Committee on Cancer, 2010.

7 Hoopes D, Tann M, Fletcher J, et al. FDG-PET and stereotactic body radiotherapy (SBRT) for stage I non-small-cell lung cancer. Lung Cancer 2007; 56: 229-234.

8 Chen F, Matsuo Y, Yoshizawa A, et al. Salvage lung resection for non-small cell lung cancer after stereotactic body radiotherapy in initially operable patients. J Thorac Oncol 2010; 5: 1999-2002.

9 Neri S, Takahashi Y, Terashi T, et al. Surgical treatment of local recurrence after stereotactic body radiotherapy for primary and metastatic lung cancers. J Thorac Oncol 2010; 5: 2003-2007.

\section{Tracheobronchial amyloidosis: evidence for local B-cell clonal expansion}

\section{To the Editors:}

Amyloidosis is characterised by the deposition of insoluble protein fibrils in organs and tissues, which leads to organ dysfunction. Amyloidosis is classified according to the composition and localisation of fibrils. The most frequent amyloidosis is immunoglobulin (Ig)-light-chain (AL) amyloidosis. In systemic AL amyloidosis, the fibrils are derived from circulating monoclonal light chains that are usually produced by intramedullary clonal plasma cells. Localised AL amyloidosis is most often identified in upper respiratory, urogenital and gastrointestinal tracts, in the skin and in the orbit. In such 